Artículos científicos 


\section{Introducción a la labor investigativa en pregrado}

Dr. José Carlos Monzón Fuentes ${ }^{1}$

\section{Resumen}

La labor investigativa debe ser considerada como un aspecto fundamental del quehacer cotidiano del profesional de la salud. La capacidad de investigar se espera que sea inherente a cualquier profesión relacionada con la salud, especialmente para alcanzar el máximo nivel de profesionalismo y beneficio para el paciente. A continuación, se presenta una reflexión acerca de la investigación en pregrado y los desafíos y oportunidades del estudiante y del profesor con respecto a esta labor, en el contexto universitario. Como conclusión, debemos resaltar la labor que debe tener un mentor y que en términos generales se prefiere a quien ha sido mentor dado que supone haber sido expuesto a una ruta muy similar durante su preparación profesional. Asimismo, el estudiante debe aprovechar situaciones inherentes al contexto en el que se encuentra para obtener provecho máximo a las labores de investigación y que puedan servir para impulsar su carrera profesional, particularmente en el ámbito académico.

Palabras clave: investigación científica, pregrado, mentoría

\section{Abstract}

The investigative work must be considered as a fundamental aspect of the health professional daily work. The ability to investigate is expected to be inherent in any profession related to health, particularly because it is desired to achieve the highest level of professionalism and benefit for the patient. Here, we present a reflection about undergraduate research and the challenges and opportunities of the student and the professor regarding this issue within a university context. As a conclusion, we must highlight the work that the mentor must have and that in general terms a mentor is preferred since he or she assumes to have been exposed to a very similar route during his professional preparation. Likewise, the student must take advantage of situations inherent to the context in which he/she finds himself/herself so as to take maximum advantage of the research work and that can serve to boost his professional career, particularly in the academic field.

Keywords: Scientific research, graduate, mentorship

'Director del Instituto de Investigación y Estudios Superiores en Ciencias de la Salud (lecis), en la Universidad Rafael Landívar. 


\section{Introducción}

En términos generales, la labor investigativa en el ámbito académico se puede clasificar en dos tipos: la investigación formativa y la investigación creativa, tal y como se describe en la Agenda de Investigación y Proyección de la Universidad Rafael Landívar (1). La investigación formativa enseña al estudiante sobre el método científico y sobre los pasos para realizar investigación. La investigación creativa, en cambio, se ocupa de crear conocimiento y asume que el estudiante ya conoce los pasos para investigar y entonces propone resolver algún problema de investigación específico, asegurándose de seguir los pasos del método científico. Y es así como se considera a la investigación formativa desde el punto de vista pedagógico y a la investigación creativa desde el punto de vista de generar conocimiento, función inherente de la educación superior (2).

Durante los años iniciales del pregrado, el entrenamiento en las ciencias de la salud se enfoca en la investigación formativa, con el objetivo de que al finalizar la carrera se pueda dar el salto de la investigación formativa

(i.e. aprender a investigar) a la investigación creativa. Y es que la investigación es uno de los aspectos fundamentales para el estudiante de ciencias de la salud, especialmente para el estudiante de Medicina. Durante este proceso se adquieren destrezas que facilitarán, en un futuro, la práctica profesional basada en la evidencia científica. Existe una tendencia generalizada de los programas académicos de impulsar la investigación científica a todo nivel, sin embargo, la participación de los estudiantes de pregrado es baja; según Alzate-Granados y colaboradores, solo el 7 \% de los procesos de investigación científica en Colombia son liderados por estudiantes de pregrado (3).

En términos generales, la labor investigativa del estudiante empieza desde que se reciben los primeros cursos de metodología de la investigación, los cuales tienen como objetivo brindar las herramientas básicas para acceder a la información formal, analizarla y tomar acciones con fundamento científico.

\section{La importancia de la investigación para los estudiantes} de la Facultad de Ciencias de la Salud

La medicina basada en evidencia centra su acción en la producción de conocimiento científico y apoyo de la práctica clínica a través de estudios bien diseñados y actualizados, que establezcan los estándares de la atención de salud de la actualidad. Este conocimiento que se transfiere a través de las revisiones sistemáticas y meta-análisis, supone ser mejor y más actualizado que aquel que se pueda encontrar en los libros de texto, además de estar disponible de inmediato con el advenimiento del internet (4).

La importancia de la investigación cae por su propio peso desde que se inicia la preparación profesional de cualquier estudiante de ciencias de la salud. Por ello, el estudiante debe ser capaz de identificar un problema particular, que pueda ser resuelto a través de una investigación viable. Es importante tener en mente el contexto personal del estudiante, dado que existen recursos no solo 
económicos sino en términos de tiempo, esfuerzo y energía que deberá dedicarle al proyecto de investigación para lograr construirlo sin sacrificar sus obligaciones académicas y de aprendizaje.

A pesar de estas limitaciones la participación de los estudiantes, en proyectos de investigación, puede realizarse en el ámbito académico como en una universidad, de manera que ambas partes salgan beneficiadas. Es así como un estudiante motivado puede impulsar su carrera como estudiante investigador, acompañado de una guía profesional que complemente las debilidades metodológicas o en términos de experiencia que el estudiante pueda tener.

Prácticas exitosas de estos dúos de investigación estudiante-profesor han sido reportadas en la literatura. Por ejemplo Liddell y colegios (5) describen experiencias de estudiantes de laboratorio con alta disponibilidad de tiempo con profesores con amplia experiencia investigativa pero con poca disponibilidad de tiempo como una historia de éxito, en la que la mayoría de las veces, los estudiantes muy motivados desarrollan amplias capacidades de investigación al ser guiados por miembros de la facultad.

Sin embargo, un desafío que también mencionan es hacer coincidir el cronograma de actividades académicas del estudiante con el cronograma del protocolo de investigación (5), dado que muchas veces el estudiante debe continuar con otras asignaturas, mientras que el proyecto de investigación aún está en la fase de recolección de datos.
La labor del docente o el tutor en un contexto que facilite la investigación

La investigación ha cobrado importancia, particularmente porque muchas instituciones académicas se clasifican de acuerdo con el número de publicaciones científicas de sus profesores asociados (6).

Una de las estrategias reportadas en la literatura para aumentar las capacidades de investigación ha sido a través de programas de mentoría (7). Estos programas han probado ser una intervención efectiva para involucrar a más estudiantes en labores de investigación, además de crear conocimiento útil a la sociedad. En el 2013, en Guatemala, se estableció un programa de entrenamiento en investigación con apoyo del Centro Internacional de Desarrollo e Investigación (IDRC), se identificaban a estudiantes de pregrado con pénsum cerrado para desarrollar una relación de mentoría en investigación y conducir un proyecto a lo largo de un año con muy buenos resultados; el $90 \%$ de los proyectos fueron presentados internacionalmente, y a la fecha de publicación del manuscrito el $50 \%$ ya contaba con una publicación en una revista indexada7.

Esto comprueba que el estudiante con un acompañamiento adecuado puede impulsar su carrera académica de una manera exponencial, no solo construye capacidades de investigación que no se obtienen en el pénsum de estudio de manera ordinaria, sino que además se convierte en un candidato competitivo para futuras aplicaciones académicas y profesionales.

Por otro lado, también está descrito que estas actividades de mentoría en investigación, son beneficiosas para el mentor (8). En estos programas, los mentores reportan más confianza en sí mismos en lo que respecta a acciones de mentoría, además de haber obtenido reconocimientos de desarrollo profesional durante el proceso (8). Esta labor del maestro se ve además potencializada en un ambiente en donde la producción científica (i.e. el número de publicaciones) de los mentores es recompensada adecuadamente a través de promociones, incentivos salariales, entre otros (4), por lo que es una relación donde todas las partes se ven beneficiadas y que se debe estimular.

\section{Oportunidades y desafíos para el estudiante del pregrado}

En el contexto de Guatemala, un país catalogado como de bajos y medianos ingresos económicos (LMICS por sus siglas en inglés) (9), existe un abanico de posibilidades de investigación debido a la falta de datos en muchos aspectos de salud. Además, por ser un LMIC, los estudiantes pueden aplicar para obtener acceso a manuscritos científicos sin costo a través de plataformas como Hinari (10), por lo que se deben aprovechar estas plataformas para realizar búsquedas de los temas a estudiar. Otra oportunidad importante para realizar protocolos de investigación es que el Ministerio de Salud Pública y Asistencia Social, a través de su plataforma electrónica del Sistema de Información Gerencial de Salud, SIGSAweb (11), se puede acceder a bases de datos ordenadas y clasificadas de acuerdo con nuestras necesidades de investigación. Estas bases de datos son 
particularmente importantes para protocolos de investigación de diseño transversal, los cuales, a juicio del autor, son los más recomendables para los estudiantes de pregrado. Estos estudios producen información importante, son cortos de realizar y relativamente baratos (11).

Entre los desafíos del estudiante de pregrado al realizar investigación se encuentra la falta de investigadores y mentores que orienten, los cuales han probado ser instrumentales en el desarrollo de la carrera académica de los estudiantes (5). Sin embargo, será tarea del estudiante indagar alternativas ya sea dentro de la institución académica en que se encuentra o fuera de ella, incluso atreverse a buscar en otros países y que sea a distancia.

Estudios sobre destrezas de investigación en LMIC (12), también han identificado que otra barrera es la falta de capacidades de investigación. Sin embargo, existen recursos en internet como la serie de metodología de investigación de Setia (13) que puede resultar muy útil en esta etapa.

Finalmente, vale la pena mencionar que aún con estas limitantes, el estudiante de pregrado tiene un brillante futuro en el ámbito de investigación que cada vez es más accesible con las colaboraciones internacionales que han sido progresivamente más rápidas y fáciles de realizar. Las conexiones académicas y el networking que se puede realizar en congresos y reuniones de investigación deben ser aprovechados al máximo, para tejer una red de futuros académicos, comprometidos a entregar la mejor calidad de atención en salud basado en 24 evidencia.

\section{Referencias}

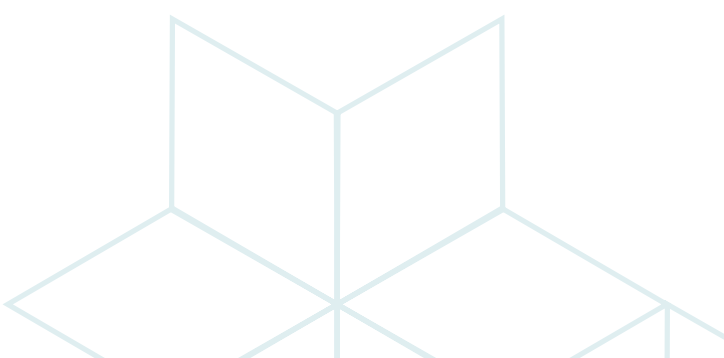

1. Gálvez J, coordinador. Agenda de investigación y proyección. Documento sintético de los programas de investigación científico-críticos. Guatemala: URL: Editorial Cara Parens; 2016. p. 15.

2. Restrepo BG. Investigación formativa e investigación productiva de conocimiento en la universidad. Nómadas. 2003;18(195):196.

3. Alzate-Granados JP, Caicedo-Roa M, Saboya-Romero DM, Pulido JC, GaitánDuarte HG. Participación de estudiantes de pregrado de medicina en revistas médicas y académicas colombianas indexadas en Publindex, categorías A1 y A2, en el período 2009-2012: revisión sistemática de la literatura. rev.fac.med. [internet]. 2014 enero [citado en 2019, mayo 16]; 62(1):9-15. Disponible en: http://dx.doi.org/10.15446/revfacmed.v62n1.4365

4. Bhargava K, Ghargava D. Evidence Based Health Care. Sultan Qaboos University Medical Journal. 2007;7(2):105-107.

5. Liddell P, Heuertz R. Students as Vital Participants in Research Projects. Clin. Lab. Sci. 2011; 24:66-70.

6. Waller KV, Karni KR. Scholarly activities of the most productive CLS faculty and schools in the USA. Clin Lab Sci. 2010;23:175-9.

7. Barnoya J, Monzon J, Colditz G. Increasing Chronic Disease Research Capacity in Guatemala Through a Mentoring Program Can J Public Health. 2013;104(5):e427-e432.

8. Feldman MD, Steinauer JE, Khalili M, Huang L, Kahn JS, Lee KA et al. A mentor development program for clinical translational science faculty leads to sustained improved confidence in mentoring skills. Clin Transl Sci. 2012;5(4):362-67.

9. Banco Mundial [internet]. Citado [2019/05/17]; Disponible en: https:// datahelpdesk.worldbank.org/knowledgebase/articles/906519-world-bankcountry-and-lending-group

10. World Health Organisation. Hinari: Access to research for health programme. Geneva: World Health Organization, 2017. [internet]. Cited [2019/05/17]; Disponible en: https://www.who.int/hinari/en/

11.Ministerio de Salud Pública y Asistencia Social. [internet]. Citado [2019/05/17]; Disponible en: https://sigsa.mspas.gob.gt/formularios/category/14-datos-desalud-ano-2009.html

12. Franzen S, Chandler C, Siribaddana S, Atashili J, Angus B, \& Lang T. Strategies for developing sustainable health research capacity in low and middle-income countries: a prospective, qualitative study investigating the barriers and enablers to locally led clinical trial conduct in Ethiopia, Cameroon and Sri Lanka. BMJ open 7(10), e017246. https://bmjopen.bmj.com/ content/7/10/e017246

13. Setia M. S. (2016) Methodology Series Module 3: Cross-sectional Studies. Indian Journal of Dermatology, 61(3):261-264. Doi:10.4103/00195154.182410. pp. 262-63. 